\title{
An Optimized Salahaddin University New Campus IP-Network Design using OPNET
}

\author{
Ammar. O.Hasan \\ Dept. of Information Technology \\ Salahaddin University-Hawler \\ Erbil, Iraq
}

\author{
Raghad Z.Yousif ,PhD \\ Dept. Applied \\ Physics-Communication \\ Salahaddin University-Hawler \\ Erbil, Iraq
}

\author{
Rakan S.Rashid \\ Bardarash Technical Institute \\ Duhok Polytechnic University \\ Duhok, Iraq
}

\begin{abstract}
Salahaddin University is the oldest and the biggest university in Kurdistan region. It involves 14 colleges and 3 academic centers. The new university campus that will be established on an area of $10 \mathrm{~km}^{2}$ provides a challenge of designing, efficient and robust networking infrastructure due to increased demand on data and data processing applications like FTP (File Transfer Protocol) which is a vital protocol in an academic environment. At access layer (college level) Wireless Local Area Network (WLAN) is employed to provide Wi-Fi to the end user for ultimate mobility. At backbone level four scenarios have been proposed and tested for a proposed university campus. These scenarios used to connect each college Wi-Fi router to Cisco core switch (6509). The first scenario uses optical fiber cable 1000Base-LX (Gigabit- Ethernet), while in the second scenario the Virtual Local Area Network (VLAN) based core switch is used to connect (Gigabit- Ethernet) cables. The third scenario uses FDDI (Fiber Distributed Data Interface) technology. In the fourth scenario, a combination of the VLAN based core switch and FDDI is presented. In the four scenarios, the core switch is connected to the main router Cisco (7507) which connects the campus network to the cloud. The network performance and behavior have been studied by calculating network load throughput and delay. The system has been implemented using OPNET (Optimized Network Engineering Tool) simulator modular 14.5. The simulation results show that the fourth scenario gives minimum delay while maximum data transfer (throughput) is achieved by the fourth scenario.
\end{abstract}

Keywords-Fiber Distributed Data Interface (FDDI); Optimized Network Engineering Tool (OPNET); File Transfer Protocol (FTP); Virtual Local Area Networks (VLAN); campus network

\section{INTRODUCTION}

Today, information technology is strategically important to the goals and ambition of the business and academic enterprises, government and academic institutions. Universities are encountering big challenges of the importance of providing faster communication services and enhancing the capabilities and skills of individuals. New challenges, such as providing an information network support the increasing demands and variegation of university issues. University Network helps universities to be more collaborative centers, which helps them to attain and implement their academic programs with higher level of knowledge for the students. University Campus network main mission is facilitating collaborative researches and building knowledge through achieving teaching goals. The network architecture, which is based on design principles of network construction is the backbone that enables the university's faculty, researchers, students, administrators, and staff to find out, learn and serve society. There are four important components in the proposed architecture, which are: Services like FTP protocol, Network Control, Core Switching and Edge Access [1]. This research presents a suggested design for Salahaddin University new campus networks model which is reliable, robust and can easily upgraded. Salahaddin University Hawler is expanded both horizontally and vertically. The vertical expansion is due to addition of new colleges and departments about 70 academic departments distributed among fourteen colleges beside the presidency and the chancellor office while the vertical expansion is due to increased number of student and academic staff which leads to growing demand on network services like FTP VoIP, etc. Thus, the huge demand on additional bandwidth and throughput cannot be achieved by traditional network design. Four scenarios have been presented and tested using OPNET simulator. The proposed network design methodology has divides the network into three layers the access layer, the distribution layer and the core layer. The core network provides the backbone for the network. The distribution layer aggregates multiple technologies from the access layer. These technologies include ISDN, DSL, cable, Ethernet, and Wireless. The access layer is the first point of communicate with the network as so it provides the required interfaces for edge devices and stations. The proposed design and modeling has submitted a comparative study based on network performance between different technologies in distribution layer like VLAN, FDDI, and Gigabit Ethernet by considering FTP and HTTP protocol at services level. The research has been classified into four sections the literature review, the description of proposed network design, the results and discussion and the conclusion.

\section{LITERATURE REVIEW}

Many researches and projects have been conducted to network modeling, simulation and verification: J. Theunis et al. [2], points out, how OPNET can be applied in designing advanced networking educational network, which leads to improving the practical skills of future network engineers. V. Hnatyshin et al. [3] examined the student and faculty usage of network applications and its influence on the Rowan university network design model. D. Akbas [4] has built both real virtual 
enterprise network model, and comparing network models and network analysis results. The effects of firewall and VPN on presented modules are studied in both real network devices and virtual OPNET environment. Ibikunle Frank [5] presents a campus network designed using multiprotocol labels switching virtual private network to increase network availability, scalability, flexibility and security. Tush et al. [6] proposed a design for industry standard hierarchal layout of campus network using different routing and switching protocols like OSPF, BGP, EIGRP. Malek N. Algabri et al. [7], had investigate and analyze the performance of MPL, and (MPLSVPN) network models for Sana'a University. Dhirendra Sharma et al. [8] discuss the efficiency of various networks commissioned on different campuses of six universities in the Western Himalayan region of India, within the framework of B-Node theory. Agueda Sofia Tavares, [1] study a reference model Architecture of University Campus Network that can be followed or adapted to build a robust yet flexible network that respond next generation requirements. Bagus Mulyawan et al. [9]. Presents a way to design campus network then implement it using top down Approach. Baek-Young Choi et al. [10] carried out in-depth outage and failure analysis of a university campus network using a rich set of both node outage and link failure data. They investigate the aspects of spatial and temporal localities of failures and outages, the relation of link failure and node outage, and the impact of the hierarchical and redundant network design on outage.

Modhar A. Hammoudi [11] propose a model for Mosul University campus which was made up two routers cisco 2600 , core switch, Cisco 6509 two servers with IP32 cloud and 37 VLANs he has investigated the possibility of applying VoIP on proposed design. Potemans et al. [12] has proposed student network design project using OPNET for the catholieke Unisiteit Leuvin the student network has been examined with the VoIP services. B. Rodiers [13] proposed a model in OPNET modeler to simulate the backbone computer network at the K.U. Leuven University in Belgium.

\section{DESCRIPTION OF PROPOSED NETWORK MODEL FOR (SUNC)}

The proposed network model for SUNC has gained the advantages of hybrid wired/wireless network models. Hybrid networks provide campus university excellent advantages in terms of speed, mobility, and security. Wireless network can be used as an extension of wired network. The estimated span area for the new campus is about $10 \mathrm{~km} 2$ this area would be shared among fourteen colleges and three academic centers, these academic centers are IT-center E-learning center and center of scientific researches and university administration (chancellor office) hence, the total number of locations is about 19 locations while in fact the academic centers are imbedded with the college campus its belongs to for example the e- learning and IT centers where included with college of science campus and research center is belongs to the presidency center. The presidency administration location is the place where the core switch and servers are mounted in that the real number of locations is abbreviated to 14 campuses and 1 administrative area. It's assumed that the distance between each college campus and the core switch is no more than $1000 \mathrm{~m}$ and consists of the following components (as shown in Fig. 1):

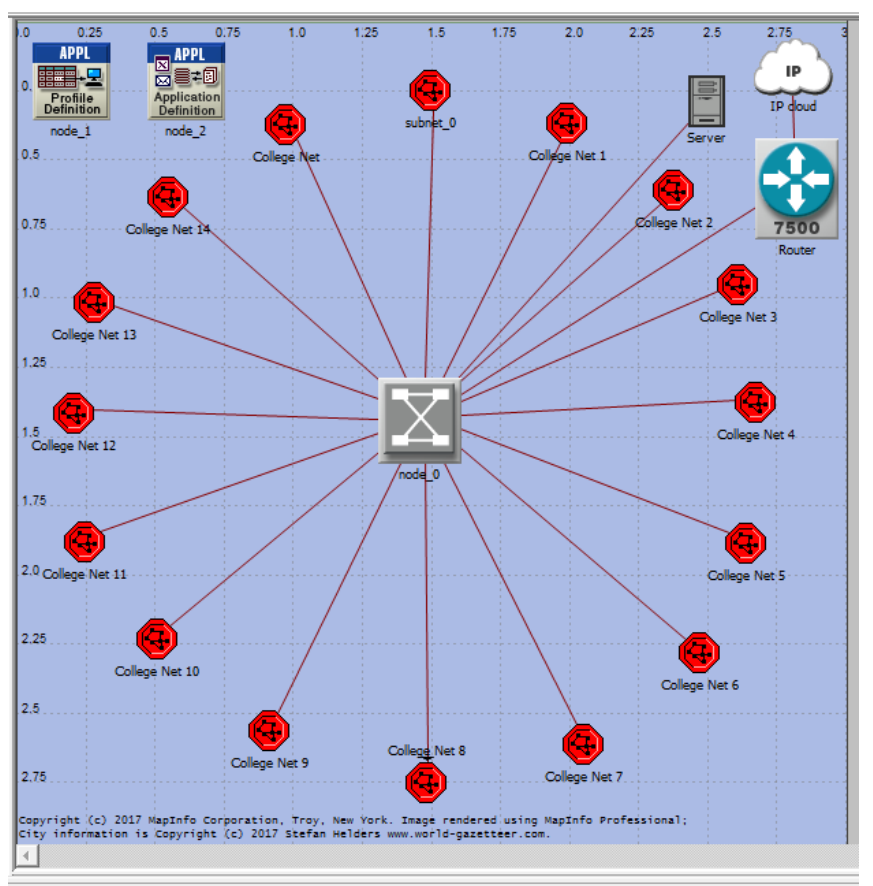

Fig. 1. The maim network model for the proposed model.

TABLE I. SPECIFICATION OF COMPONENTS

\begin{tabular}{|c|c|c|}
\hline \multirow{2}{*}{ Item } & \multirow[b]{2}{*}{ Description } & \\
\hline & & Quantity \\
\hline $\begin{array}{l}\text { Total No. of } \\
\text { Computers }\end{array}$ & Personal Mobile Computers & 241 \\
\hline Core Switch & $\begin{array}{l}\text { Ethernet Connections at the } \\
\text { CISCO Catalyst switch } 6509 \\
\text { specified data rate }(10,100,1000 \\
\text { Mbps) }\end{array}$ & 1 \\
\hline Wireless Workstation & $\begin{array}{l}\text { Personal Computers with Wireless } \\
\text { LAN card each }\end{array}$ & 240 \\
\hline Router & The Cisco device (Cisco 7507) & 1 \\
\hline IP Cloud & $\begin{array}{l}\text { Device Name: } \\
\text { eth64_s164_atm16_fr16_adv }\end{array}$ & 1 \\
\hline $\begin{array}{l}1000 \text { Basex Fiber } \\
\text { optic cable }\end{array}$ & $\begin{array}{l}\text { Sufficient for } 1000 \text { meters and } \\
\text { speed of 1000Mbps } 4 \text { Twisted } \\
\text { paired single wires }\end{array}$ & $5000 \mathrm{~m}$ \\
\hline 1000BaseX & Sufficient for 2000 meters at least & \\
\hline Wi-Fi networks & $\begin{array}{l}\text { Wireless Router distributes a Wi- } \\
\text { Fi to the mobile hosts }\end{array}$ & 16 \\
\hline $\begin{array}{l}\text { Wireless LAN based } \\
\text { router }\end{array}$ & $\begin{array}{l}\text { WLAN_Ethernet_router_adv. } \\
\text { Wlan (IEEE 802.11) }\end{array}$ & 16 \\
\hline
\end{tabular}

The table of component used in proposed design is shown in Table I.

The colleges are distributed around a central building of the university admiration (University presidency location), which is the core location by which the university traffic is switched and routed. Fig. 2 shows the proposed design hierarchy which illustrates college network topology (star topology) and how it's connected to the core switch and router in OPNET Modular 14.5.

The proposed network model is made up of two routers (Cisco 2600), core switch (Cisco6509), two servers, IP-32 
cloud (connect to the internet). All college campuses are connected to the core switch physically as shown in Fig. 3.

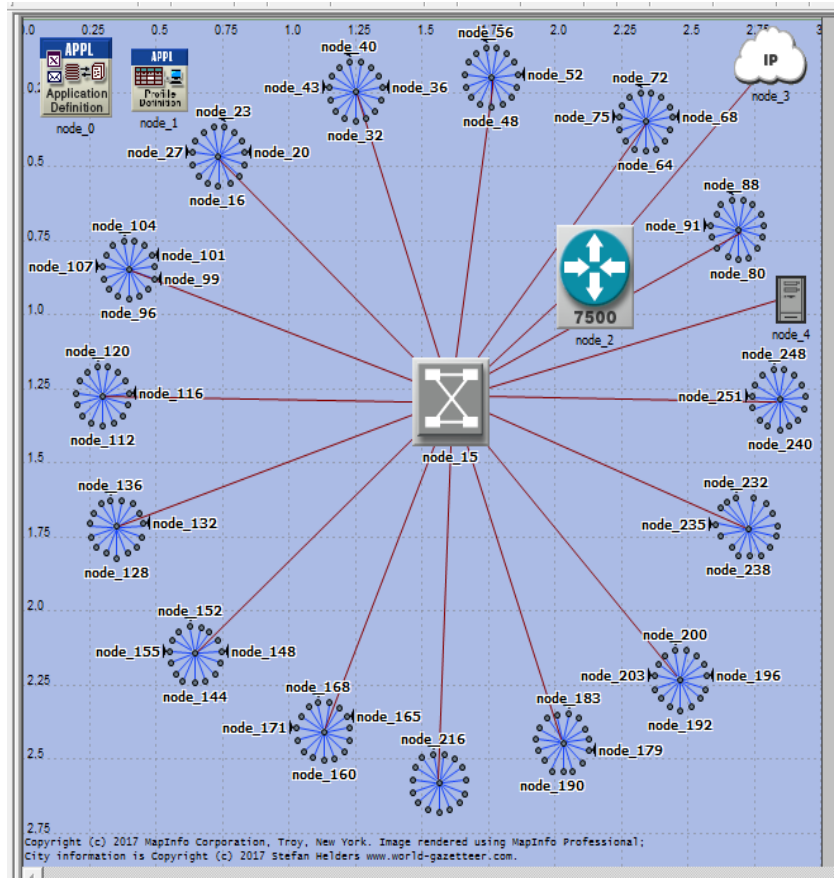

Fig. 2. SUNC network topology viewing more details in each college wireless network.

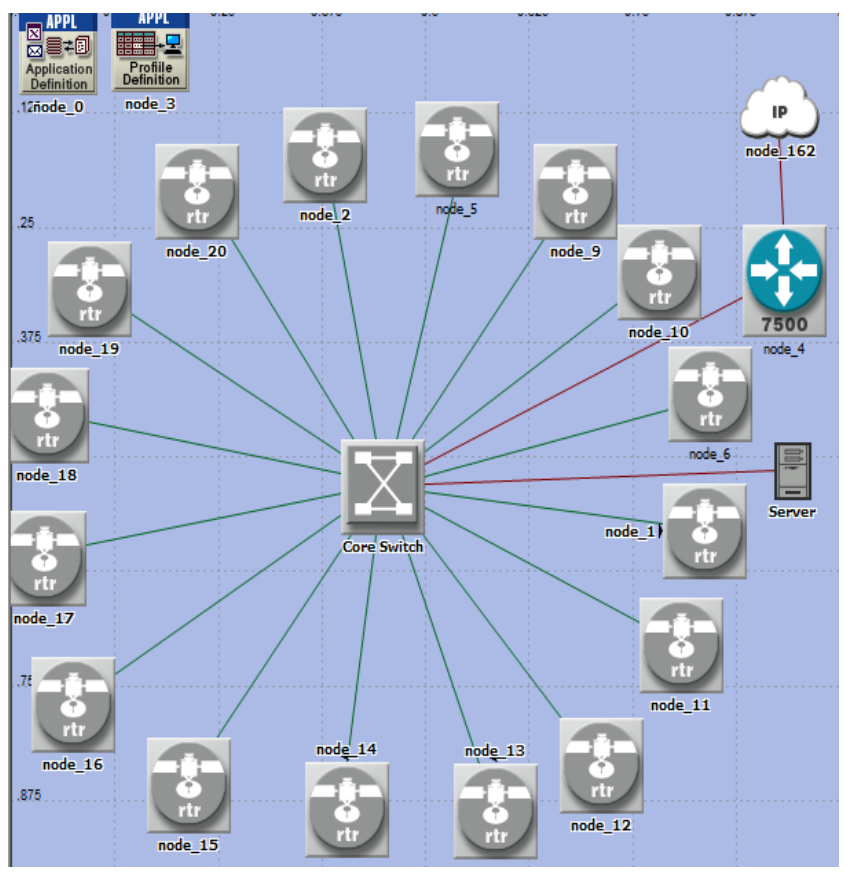

Fig. 3. SUNC network topology illustrated with Wi-Fi access point at each college center.

While inside each college campus as shown in Fig. 4 the workstations are connected throw an access point as indicate in Fig. 4. The four proposed scenarios keep same network hierarchy with different logical configuration and physical connection between campus access point and core switch. At campus level, a wireless network connection is selected to add more flexibility to the end user and to achieve full mobility needed with the modern network connected devices and equipment like (mobile and tablets).

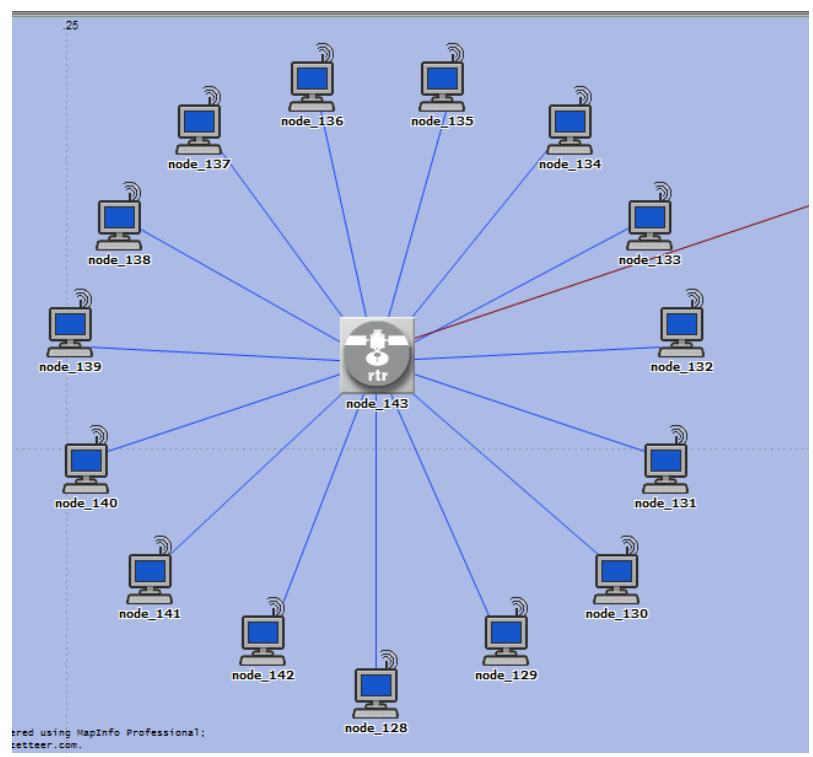

Fig. 4. SUNC network topology at access layer (each college).

In first scenario, the interconnection between the core switch and college campus access point is done by using fiber optic cable 1000Base X. the second scenario use the same previous Cable in addition we configure the network as unique VLAN. The VLAN is used to define logical units to those people of the same administrative service, research group, classrooms, etc. This gives the availability to the users of the same unit to connect to the network from geographically distributed locations spread at remote areas inside the university [11]. After connecting the nodes, their configuration must be selected by editing the attribute of each node. A subnet was created for VLAN (Virtual Local Area Networks). In the proposed VLAN the ports were configured as 'access' ports except the port connected to the core switch should be 'trunk' to allow access to internet through the trunk port in the core switch. To ensure proper connection between the computers in the network, each computer was addressed with an IP of class $\mathrm{B}$, with the subnet mask (255.255.255.0). This subnet mask, in turn, allowed 254 subnets. Each subnet has to provide them with an internet access. IP32 cloud which is one of the OPNET facilities was added to connect the network to the internet. A router links between the core switch and the IP32 cloud. The router must have an IP address for all the connected ports. The port facing the core switch was set with an IP address of the same subnet used for VLAN, and the port facing the internet was given an IP address of the subnet between the internet itself and the router. In third scenario, the FDDI (Fiber Distributed Data Interface) technology is employed to connect logically and physically each access point to the core switch. FDDI is a network technology that supports 100 Mbps transmission rate for up to 500 communicating stations configured in a ring or a hub topology. FDDI was developed by the American national standard institute (ANSI) X3 T9.5 committee in 1987. It uses fiber optic cable up to $200 \mathrm{~km}$ in length (single ring) in a LAN environment, in a dual ring 
topology, maximum distance is $100 \mathrm{~km}$ in the fourth scenario a hybrid technology between second and third scenario is presented which is VLAN based FDDI in that the logical configuration is based on VLAN configuration while the interconnections have been connected by FDDI Technology. The four proposed scenarios are tested and their performance are investigated using network common quality of service parameters like network delay, and throughput for FTP service defined on a main server.

\section{RESULTS AND DISCUSSION}

After creating the network model, the simulator has to test the performance of proposed network scenarios. Table II below summarize the scenario code, details and curve color in the OPNET graphs.

The profile name of FTP application was appended to some computers and specified the FTP server as their destination. This was done to test the traffic to/from these computers and the FTP server. In this work, FTP service besides some focus on HTTP services have been made. Fig. 5 and 6 show the responses of the network model of FTP test for each scenario. As can be seen in these figures, the responses had zero values at the beginning, and then were increased because the network should have enough time to identify its components, this might take few seconds.

Fig. 5 shows the average traffic received for the FTP service in SUNC network for all scenarios. It's clear that the minimum average traffic received is attained by the fourth scenario, whereas the comparable results have been observed by other scenarios.

Fig. 6 shows the FTP Traffic send in (byte/sec) through the network, it's clear that the fourth and second scenarios appear to utilize the network bandwidth rather than second and third scenarios.

TABLE II. SCENARIOS DETAILS WITH CURVE COLOR

\begin{tabular}{|l|l|l|}
\hline $\begin{array}{l}\text { Scenario } \\
\text { Order } \\
\text { Number }\end{array}$ & Scenario Details & Curve Colour \\
\cline { 2 - 3 } Scenario 1 & 1000BaseX without VLAN & Green \\
\hline Scenario 2 & 1000BaseX with VLAN & Blue \\
\hline Scenario 3 & FDDI without VLAN & Red \\
\hline Scenario 4 & FDDI without VLAN & Cyan \\
\hline
\end{tabular}

Fig. 7 shows the average HTTP object response time in seconds It's obvious that the fourth scenario records fastest response time as compared to other scenarios which give compatible results.

Fig. 8 shows the HTTP traffic received. It's obvious that the minimum HTTP traffic received is by the fourth scenario which gives compatible results.

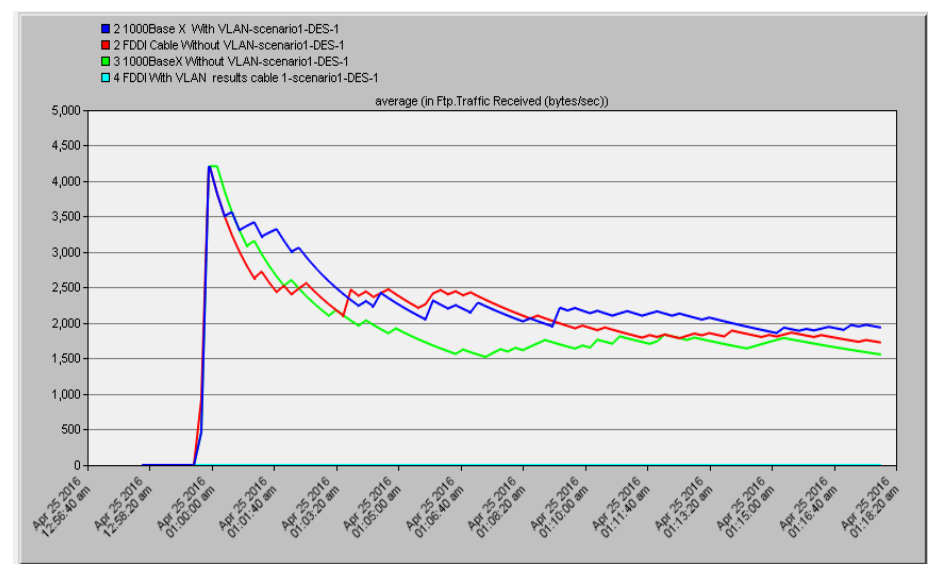

Fig. 5. SUNC average traffic received (FTP Service) in byte/sec.

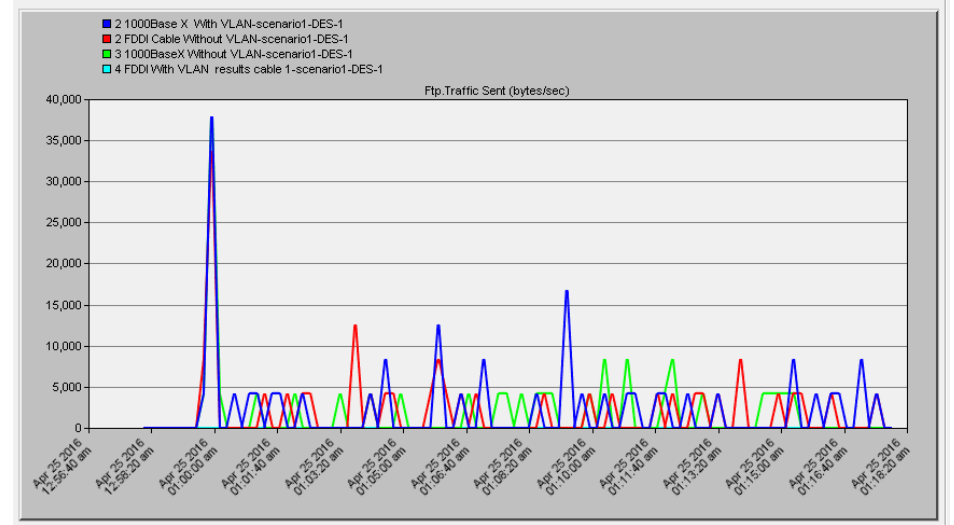

Fig. 6. SUNC network Traffic sent (FTP Service) in byte/sec.

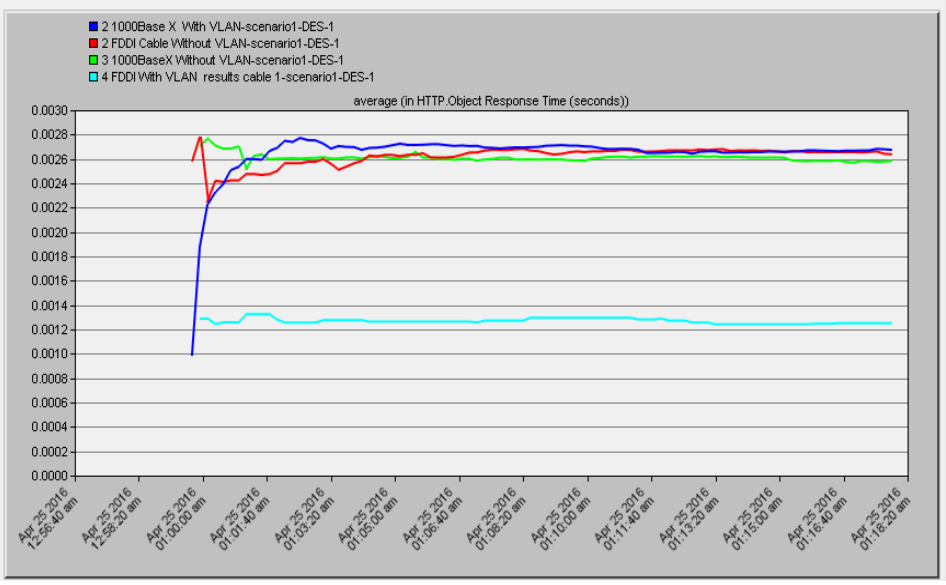

Fig. 7. SUNC network the HTTP object response time in (sec).

From Fig. 9 below which shows the HTTP service Response Time in sec, the fourth scenario gives shortest response time as compared to another scenario, while the $3^{\text {rd }}$ scenario gives relatively longest response time. 


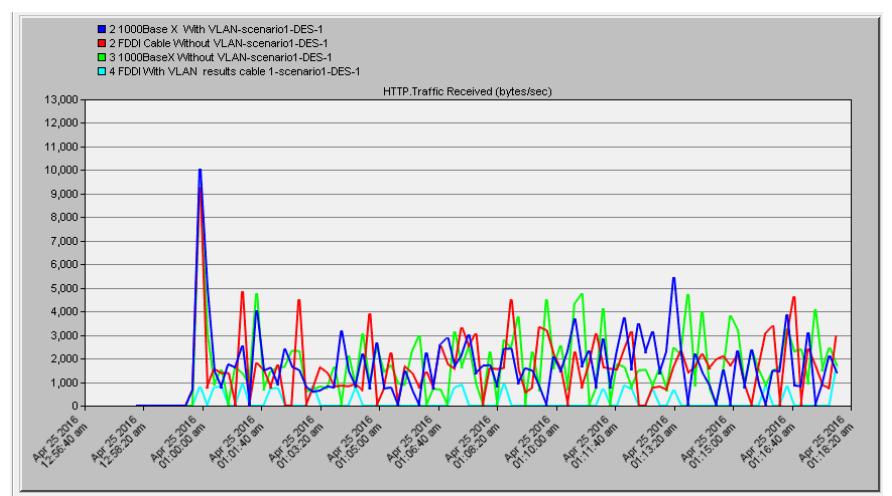

Fig. 8. SUNC network the HTTP traffic received in (byte/sec).

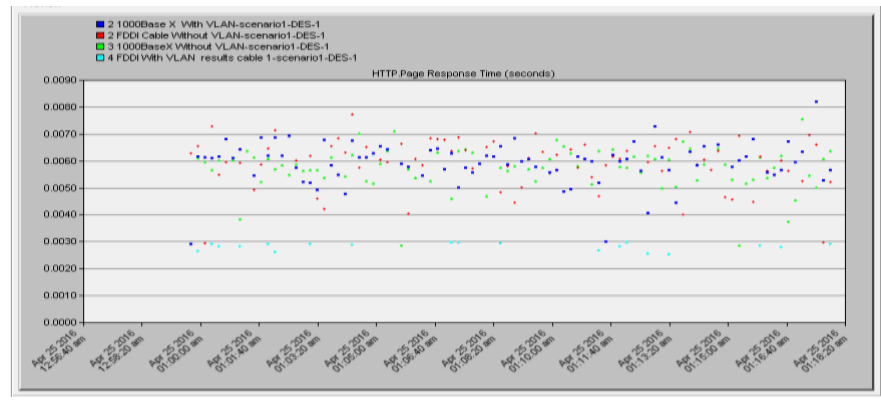

Fig. 9. SUNC network the HTTP page response time in (sec).

Fig. 10 depicts a comparison based on Traffic received (packet/sec) for the VLAN based scenarios (second and fourth scenarios). This comparison is made to investigate the performance of these two scenarios. The fourth scenario outperform the second scenarios by reducing the amount of traffic received, this due to the reliability of FDDI technology.

Fig. 11 shows the average wireless LAN Media access delay. The fourth scenario gives minimum LAN medium access delay in sec. While first second and third scenarios give comparable results.

In Fig. 12 which depicts the Ethernet delay calculated during the heavy load period, it's clear that the third scenario, shows maximum delay about $0.000085 \mathrm{sec}$, while the fourth scenario gives minimum delay about 0.000008 .

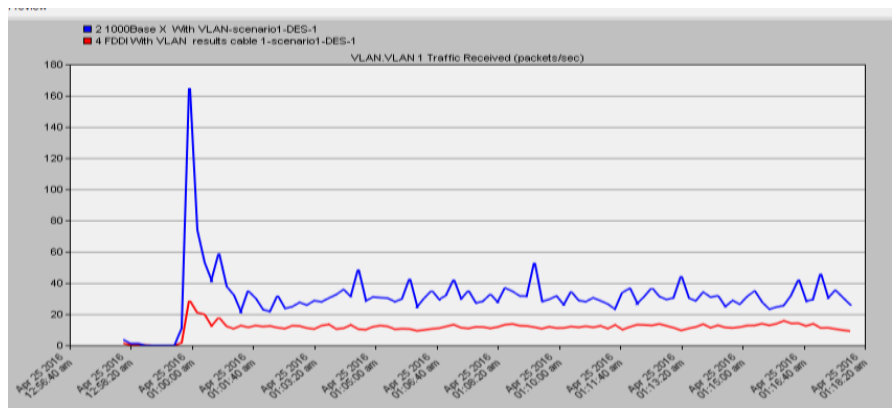

Fig. 10. Traffic received (packet/sec) for fourth and second scenario.

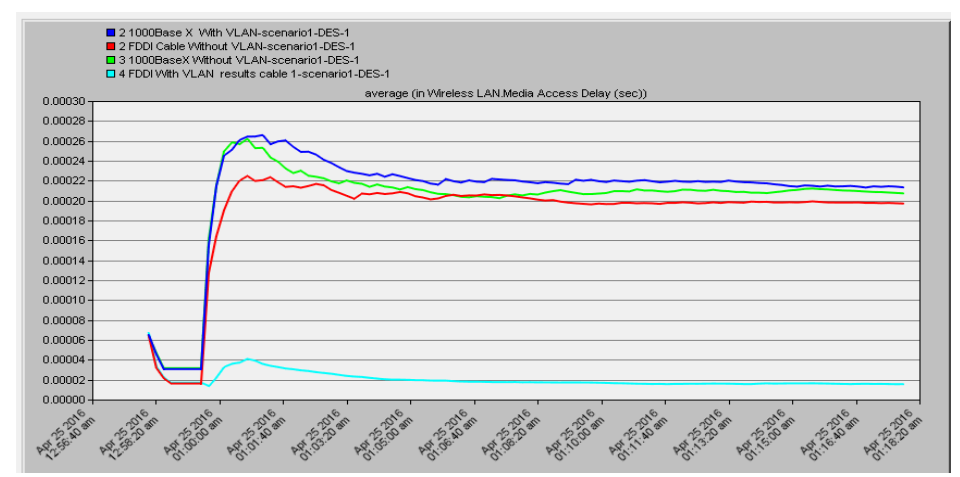

Fig. 11. Average Wireless LAN Media access delay.

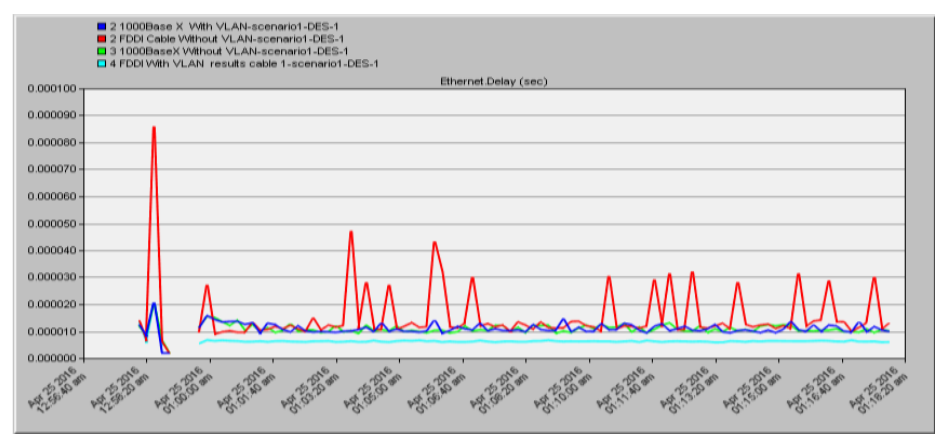

Fig. 12. SUNC network ethernet delay.

\section{CONCLUSION}

This paper depicts the ability of OPNET simulator tool in preparing and simulating a model of the new campus computer network at Salahaddin University. As can be seen from the figures of FTP, HTTP, wireless LAN and Ethernet delay, that the responses had zero values at the beginning, and then began to increase because the network should take sufficient time to recognize and identify its components and devices; therefore, this may take few seconds. Four scenarios have been proposed and discussed, based on traditional and new technologies. The fourth scenario which combine VLAN and FDDI technologies show superior properties in term of access delay, Ethernet delay and HTTP with FTP response time and load. While other scenarios give comparable results. In the future a real implementation of the fourth scenario will support the results obtained by this research paper.

\section{REFERENCES}

[1] Águeda Sofia Monteiro Tavares," Network Architecture for University Campus Network", Master thesis, in Communication Engineering of Chongqing University, Chongqing China, May 2011.

[2] J. Theunis, B. Van den Broeck, P. Leys, J. Potemans, E. Van Lil, A. Van de Capelle, "OPNET in Advanced Networking Education", OPNETWORK 2002, Washington D.C., USA.

[3] V. Hnatyshin, A. F. Lobo, P. Bashkirtsev, R. Domenico, A. Fabian, G. Gramatges, J. Metting, M. Simmons, M. Stiefel, "Modeling a University Computer Laboratory using OPNET Software", Computer Science Department, Rowan University, New Jersey, USA, 2006.

[4] D. Akbas, Modelling and Analysis of an Enterprise Network and its Security Structures, Master Thesis, Haliç University, Department of Computer Engineering-Management Information Systems Program, July 2010. 
[5] Ibikunle Frank, Oloja Segun, Esi Phillips, "Campus Network Virtualization Using Multiprotocol Label Switching Virtual Private Networks (MPLS-VPNs)", International Journal of Applied Information Science, USA, Vol.5, No.8, pp.1-9, June 2013 (ISSN: 2249-0868).

[6] Tushar Krishna, A.Banerjee," Automation and Analysis of Enterprise Campus Network Design", PARipex, Indian journal of researches, Vol 5,issue 5,May 2016.

[7] Malek N. Algabri, Sharaf A. Alhomdy, Gamil Alselwi, Ameen A. M. Alowiri, Naif Al-Sharaby "Performance Analysis Of IPV6 Over MPLls \& MPLS-VPN For Sana'A University", JISET Vol 3, Issue 5, Pages 165-169, May 2016.

[8] Dhirendra Sharma, Vikram Kumar, Marco Zennaro, Vikram Singh "A Study of Efficiency- Campus Networks in Western Himalayan Universities of India", Workshops of International Conference on Advanced Information Networking and Applications, pp 751-756,2011.
[9] Bagus Mulyawan, "Campus Network Design and Implementation Using Top down Approach", Proceedings of the 1st International Conference on Information Systems for Business Competitiveness (ICISBC) 2011.

[10] Baek-Young Choi, Sejun Song, George Koffler, Deep Medhi, "Outage Analysis of a University Campus Network," IEEE 16th International Conference on Computer Communications and Networks (ICCCN), (Invited Paper) Honolulu, Hawaii, August 2007.

[11] Modhar A. Hammoudi," Building Model for the University of Mosul Computer Network Using OPNET Simulator", Tikrit Journal of Eng. Sciences, Vol.18, No.2, pp.34-44, June 2011.

[12] J. Potemans, J. Theunis, M. Teughels, E. Van Lil, A. Van de Capelle, "Student Network Design Projects using OPNET", OPNETWORK 2001, 2001.

[13] B.Rodiers, "Analysis and Simulation of The K.U.Leuven- network", Master Thesis in Electronics Engineering, K.U.Leuven, Belgium,June 2002. 